\title{
Harmonised consent in international research consortia: an impossible dream?
}

\author{
SUSAN E. WALLACE AND BARTHA M. KNOPPERS ${ }^{1}$
}

\section{Introduction}

It is well recognised that it can be difficult for researchers to ensure that proper consent provisions are in place for their research project. If this can be difficult for those conducting a single site study, those difficulties can be compounded when that study becomes a member of an international scientific research consortium. These consortia bring together often diverse groups of researchers who, while working on a common topic, may represent different countries, cultures and scientific methodologies. Harmonising consent information and processes across these studies can be a complicated task. At the local level, participants need to be informed of the details of the study to which they are being recruited, and informed of the implications of the study's inclusion in the consortium. Likewise, the international consortium needs to make certain that member studies have met appropriate consent requirements so that participants' samples and data can be shared with and used by the consortium for agreed purposes. A considerable amount of time and effort is needed to ensure an international consortium is running consistently across its constituent parts. And as the consortium grows, so do the complexities of dealing with the different regulatory and cultural norms of its members, while staying within the organisational requirements of the consortium itself. But a level of consistency, in terms of consent to use of data and samples, must be achieved across the consortium members in order for its work to proceed while respecting the consent provisions agreed to by participants. The question of how to arrive at this benchmark is a vital one. This paper will present issues raised as a result of an examination of consent materials used by International Cancer Genome Consortium (ICGC) members.

\section{The rise of international consortia}

The number of international consortia creating resources for the scientific community is increasing, yet they differ in how data and samples are collected, stored and disseminated. The International HapMap Project and the 1000 Genomes Project, for example, created open and public resources of anonymous genotype data. ${ }^{2}$ Others collect identifying data and samples. The Type 1 Diabetes Genetics Consortium (T1DGC), ${ }^{3}$ a project identifying genes related to this disease, used a centralised system where samples and personal data were collected, coded and stored in regional network repositories as well as in a central repository in the United States. T1DCG member projects had to comply with applicable US national regulatory requirements. ${ }^{4}$ The Malaria Genomic Epidemiology Network (MalariaGEN) is a global research network that was established to investigate genomic variation in humans on the biology and pathology of malaria and has partner institutions in 17 countries. ${ }^{5}$ In this latter network, after coding data and samples to protect the confidentiality of 
participants, researchers "...contribute [them] to a central repository of DNA samples and a central database of core phenotypic data for each Consortial Project."

In $2008,{ }^{7}$ the ICGC “....was launched to coordinate large-scale cancer genome studies in tumours from 50 different cancer types and/or subtypes which are of clinical and societal importance across the globe." ${ }^{.8}$ Founders agreed that no one country could conduct the project on its own but that multiple initiatives would unnecessarily duplicate efforts. Acting in a concerted fashion gives ICGC researchers opportunities to harmonise data sets, leading to accelerated dissemination of data and analytical methods to the user community. ${ }^{9}$ As of December 2011, 39 projects in 13 jurisdictions are active under the ICGC banner. ${ }^{10}$ These include both prospective studies and retrospective uses of existing samples and datasets. The ICGC is continuing to bring in new members and member studies are publishing the results of their work within the ICGC. ${ }^{11}$

Datasets from ICGC member projects are also now accessible. Unlike T1DGC and MalariaGen, the ICGC chose a federated system where samples would be kept by the local project and data derived from samples and other associated data would be submitted and made available through open and controlled access databases. Open access data is publically available data from the member projects which can be searched via the ICGC Data Portal to find, for example, mutations on chromosome 7 found in participants with malignant melanoma. Participant data is coded and even if the elements in this database (i.e., gender, cancer pathology) were aggregated, they would not create a dataset that is unique to, and thus could identify, an individual. On the other hand, Controlled Access Datasets contain “...composite genomic and clinical data that are associated to a unique, but not directly identified, person." International researchers need to apply to and be approved by the ICGC Data Access Compliance Office (DACO) in order to be given access to controlled data.

International consortia such as ICGC are virtual in nature and although 'management' offices are located in one country, the consortium itself is not bound by the legislation of any one country. In the case of ICGC, the Secretariat is hosted by the Ontario Institute of Cancer Research in Toronto, Canada. Decisions on how the consortium will work, however, are made by the ICGC Executive Committee, which comprises representatives from funding agencies based in the countries of participating projects. This bottom-up approach grounds decisions at the level of the member projects, but does not base them on any one set of national laws. Decisions are negotiated between the members, who use international instruments, ${ }^{13}$ in addition to applicable national ones, ${ }^{14}$ as guidance when creating policies and seeking best practices. However, not having a single national regulatory structure in place can potentially leave gaps in oversight. If no top-down compliance mechanism exists, an international consortium has to trust that its member studies are being conducted in accordance with local regulations, national and international law and ethics policies, and have appropriate institutional oversight. In addition to that collegial trust, the international consortium itself needs to have policies and guidelines in place, with appropriate governance 
systems, for its oversight and to assist it in seeking the harmonisation of working practices that will enable it to proceed.

But these working practices always need to take into account that member studies represent different countries and cultures. ${ }^{15}$ Consent form language and the consent process itself will also vary from project to project and is necessarily culture- and context-dependent. ${ }^{16}$ Member studies may also use varying scientific methodologies; regulatory and ethical review procedures for seeking consent for a prospective research study will differ from those for use of existing samples consented for different research purposes. But even accepting these differences, the area of informed consent is one vital area in which there must be consensus, or at a minimum, interoperability between compatible approaches and core elements, in order that participants are informed about the research in which they have agreed to participate.

Recent reports have highlighted how unclear consent materials and procedures can delay or even prevent data and samples being used in research. ${ }^{17}$ For example, the cell lines reported by Conrad and colleagues in their 2008 paper, and expected to be available to researchers, had not been consented properly. ${ }^{18}$ Consent forms did not allow the cells to be retained after three years and they had to generate new ones, at the expense of time, funding and dissemination to the scientific community. ${ }^{19}$ As a Nature editorial notes about this case, "...the act of research and the ethics relating to a field of study are not always in synchrony, which can introduce unfortunate - and unacceptable - restrictions. ${ }^{, 20}$ Such restrictions can block the progress of a member research study and seriously impact the work of the consortium. In order to try to avoid such inconsistencies, consent needs to be synchronised and harmonised, as far as possible, across consortium members.

\section{Examination of ICGC member consent materials}

In Spring 2010, the ICGC Ethics and Policy Committee (EPC) decided to examine how member projects had incorporated different core and suggested ICGC guideline elements ${ }^{21}$ into their consent materials. As well, the EPC was interested in knowing whether projects were using the ICGC Research Study Model Consent Brochure (Prospective Research) written by the EPC. (Supplemental data) Consent materials from ten projects, representing seven countries, were submitted to the EPC and reviewed. Materials from retrospective projects, having received an ethics committee waiver in order to participate, were not submitted and reviewed, as the original consents would not have mentioned ICGC. Only the language explaining scope of research, international data-sharing and return of individual research results was examined. This was due to the time and expense that would have been incurred to commission full and validated translations. Instead, where materials were in languages other than English, one of us (SW) translated the appropriate sections relating to these three topics. These translations were returned to a representative of the member project, who was asked to confirm that the translation was accurate. Four studies (from three countries) had used the model consent materials, designed to provide the keys points ICGC had decided needed to be included. These were new 
studies specifically formed to participate in the ICGC. The other six projects had revised existing consent materials to meet the requirements of ICGC participation.

We found that exact ICGC language was not used by every project. For example, as a core principle, consent materials must state that data and samples will be used for cancer research. However, as it is unclear where this research will lead in the future, the ICGC guidelines suggest that researchers be "...encouraged to seek the broadest level of consent that is appropriate at the local level; e.g., 'cancer and related research; cancer and other disease-related research." "22 All materials made it clear that the research was into cancer. But it was difficult to determine from the consent forms themselves exactly how far the consent extended beyond that point. This could be an issue when requests for data are received. Similarly, coded data from the member studies is put into open and controlled access databases that can be accessed by approved researchers around the world. Without consent for this, studies that do not ask for consent to such sharing cannot submit their data to the ICGC, thus negating the benefits of having that project as a member of the consortium. The review of the consent materials showed that that there might be ambiguities in the consents possibly hindering the sharing of the data when requests for access were received.

In response, the ICGC Executive Committee decided to approach member projects to clarify that their datasets were consented in line with ICGC requirements on scope of research and international data-sharing or that they had obtained an acceptable ethics review committee waiver of consent. When joining ICGC, member studies were asked to agree to the ICGC policies, so adherence to other policy points was not reconfirmed. But it was decided that these two areas were sufficiently vital to the success of the ICGC that they should be confirmed independently. Unlike other consortia, ${ }^{23}$ the Executive chose not to require member projects to translate their consent forms in order to check compliance. Instead, the Executive asked for written clarification and confirmation on these two points from member studies with data ready for inclusion in ICGC databases as of Summer 2010 (Table 1). In this way, any potential ambiguities could be found and resolved, allowing data to be disseminated in accordance with ICGC policies and participants' consent.

Table 1: Questions to ICGC Project Leaders confirming the scope of research of their dataset and the ability to share that data internationally

\begin{tabular}{|l|l|}
\hline Scope of Research: & Yes/No \\
\hline $\begin{array}{l}\text { 1a. Is your dataset consented to be used for any approved biomedical } \\
\text { research? }\end{array}$ & Yes/No \\
\hline $\begin{array}{l}\text { 1b. Is your dataset consented to be used for cancer and related research } \\
\text { (as opposed to only on one specific cancer type)? }\end{array}$ & \\
\hline International Data Sharing: & Yes/No \\
\hline $\begin{array}{l}\text { 2. Is your dataset consented to be shared with and used by ICGC and } \\
\text { international researchers outside your project and country? }\end{array}$ & \\
\hline
\end{tabular}

Genomics, Society and Policy, Vol.7 (2011) ISSN: 1746-5354

(C) ESRC Genomics Network. 
At that time, seven studies had submitted data (available in the ICGC Open Access database; no controlled data was then available); this included both prospective studies whose consent materials we had reviewed and retrospective studies. All confirmed that their datasets were consented for any approved biomedical research (a broader scope of research than requested from studies) and international data-sharing. Member projects submitting data to the ICGC in the future will also be asked to confirm that their consent materials allow international data-sharing and to clarify the scope of permissible research.

While admittedly covering only a limited number of studies, our research revealed potential problems and prompted action to deal with them. It also confirmed to us the importance of some of the processes undertaken by the ICGC when setting up the consortium, lessons that can be learned by other international consortia. First, it confirmed that international consortia need to determine core consent elements upon which all members agree and these need to be either in the consent materials or approved via an ethics committee waiver. Second, beyond the core elements, consortia need to have flexible guidelines in order to accommodate the differing ethical, legal and cultural norms of their members. Third, model consent language can help aid consistency across consents. Fourth, ambiguities and inconsistencies should be expected even with all of the above and action may need to be taken. Finally, governance procedures, such as examining ambiguities and resolving them, support the use of a broad consent for this type of research. Each of these will be discussed.

\section{Core consent elements are needed to form the backbone of the consortium}

Studies join a consortium to contribute their scientific efforts towards the common aim (e.g. the fight against cancer), as well as profit from collaborative research efforts provided by the members. In order to move these often disparate groups of researchers forward, agreement on the activities of the consortium is essential. In addition to requirements for quality standards for samples, analyses of genomic data and publication policies, the ICGC asks that projects agree to basic requirements, including core bioethical principles for prospective and retrospective research. ${ }^{24}$ These principles reflect many of the basic tenets of research ethics (e.g. the right to withdraw, the right to decide without coercion, the right to have one's medical treatment not affected by a decision to not participate etc.). As well, they state information specific to this consortium that needs to be given to potential participants. As noted, such information includes international data sharing and the fact that data and samples will be used for cancer research. One ICGC-specific process is notifying potential participants that non-identifying elements of their data (e.g., cancer pathology or age range) will be placed in an open access database and that once placed there, it cannot be withdrawn as it will be impossible to know every instance of its use. ${ }^{25}$ Another specific process is that study researchers at the local level may conduct whole-genome sequencing on the samples they collect; this data is placed in the controlled access database. Whole-genome sequencing raises many ethical issues, such as feedback of individual results, privacy and future uses of the data, ${ }^{26}$ and participants should be informed of this research use. 


\section{Flexible guidelines allow different studies to work together}

Like a human backbone, the core elements give the consortium its stability. But a consortium, just like a human, needs to be able to move freely and adjust to circumstances. While the core principles set a minimum agreement for information that will be given to potential participants, flexible guidelines can help members to adhere to their own local requirements while conforming to ICGC structures. For example, one specific area in which ICGC has found a need for flexibility at the local level is the feedback of individual results. ${ }^{27}$ It was agreed that the ICGC would not take responsibility for returning individual genetic research findings discovered by those conducting downstream research. In common with other large scale genomic data and sample collection projects, this is a research project and is not involved with individual clinical care. Research findings are not expected to have individual predictive value; general research findings will be made available on the ICGC website. ${ }^{28}$ In addition, the data shared with the ICGC is coded; researchers accessing it agree not to re-identify individuals. However, the ICGC recognises that there may be an ethical, legal and/or medical imperative to return actionable findings of clinical significance to individuals and the most logical place for this responsibility is at the local level. Unlike ICGC, the investigators recruiting participants will have in place institutional mechanisms at their disposal to ensure proper processes are followed. Therefore the ICGC guidelines state that:

Provided it is agreed at recruitment, if clinically important and validated findings emerge during the initial recruitment and screening phase, or in the early research, attempts will be made to pass this information back via the clinician, by whatever mechanism may be agreed at the local level. ${ }^{29}$

However, the ICGC has recognised that this is a rapidly moving issue; the EPC is currently conducting research asking its members about their experiences with returning results and the particular issues that they faced. Findings will be published and used to craft future ICGC policy as well as to assist the international community on this issue.

\section{Model consent materials can aide consistency across member projects}

While the ICGC needs to allow flexibility in the wording of consent materials, it cannot be flexible with the core elements agreed to by the consortium, so model consent materials were created. Model consents are being used widely as they afford researchers guidance on items of information that should be included in materials in order to ensure compliance with requirements, consistency with their fellow consortium members, or to help them follow 'best practice. ${ }^{30}$ The model consent for prospective research, based on the Public Population Project in Genomics $\left(\mathrm{P}^{3} \mathrm{G}\right)$ model templates, ${ }^{31}$ was designed for new ICGC studies and prospective studies revising their consent materials in light of ICGC participation. The ICGC Research Study Model Consent Brochure (Retrospective/Secondary Use of Research) ${ }^{32}$ aids projects seeking to re-consent participants in existing studies. Both provide model language for participating in a large-scale international consortium and can be 
modified according to the cultural and regulatory requirements of the country or institution. Like other biospecimen-based projects, ICGC consent materials need to make clear how samples are being used and what data are being made available to the consortium and the wider research community. Participants need to be told how this will happen, how their identity will be protected, what information they can expect back from the project, what ongoing monitoring of regulatory compliances are in place as well as their rights and conditions of withdrawing from the project. What must be included is context-dependent; ${ }^{33}$ a consent form for research involving a patient facing surgery differs dramatically from one that a healthy volunteer signs to participate in a research biobank. However, when these activities begin to come together - participation in a biobank where one's leftover tissue from oncological surgery will be used for future unknown research - it is important to be clear as to what information is necessary for informed consent.

In addition, consent materials can vary greatly depending on the setting. ${ }^{34}$ The ICGC models help to remind researchers of the core elements that cannot be changed and need to be conveyed to potential participants and indicate where there is flexibility. For example, the model consent confirms the policy that once data is in the open access database, it cannot be withdrawn. But it also allows some flexibility regarding whether or not samples have to be destroyed or simply not used further:

If you withdraw from the [Study], your remaining samples [will be destroyed and], the data derived from any analyses done on your samples, and other personal information will be no longer used [by the study] .... Coded data placed in the Open-Access database cannot be withdrawn, but no additional information will be added.

ICGC studies are not required to use the models, which were created as an aid and resource. While enforcement of the use of model consents might help achieve harmonised consent across the consortium, this process would no doubt involve the potential re-consent of hundreds of people, an effort most likely not budgeted for by many research studies. It would also potentially preclude the use of existing collections, which can be brought into ICGC with an ethics committee waiver. Reconsent and making existing collections unusable would delay the work of the consortium and its goal of furthering cancer research and incur added expense. In preference, ICGC suggests using the models and recognises that consent materials will necessarily vary. Model consents can also help in other ways, such as with the ethics review process. One member has stated that it not only helped them with writing the consent materials, his IRB was also very appreciative, as it helped them to understand the aims and structure of the ICGC and the requirements of local participation. $^{35}$

\section{Ambiguities and inconsistencies may still arise}

Even with model consents and guidance in place, the examination of the ICGC member consent materials showed that there are still ambiguities and inconsistencies 
across the studies. This must be accepted; as noted earlier, international consortia such as ICGC have no top-down enforcement mechanisms available to them to impose requirements on members. Even when desired by a consortium, consent materials do not always conveniently use exact words. , Wording indicating breadth of consent, such as, for example, 'for biomedical research', is open to interpretation. Two causes for this can be pinpointed. First, the consent materials were a mix of new consents written using the wording of the ICGC model consent with existing consents adapted for ICGC use. This naturally introduced a lack of uniformity. The second issue is one that is faced my research studies that are part of a larger study creating ongoing resources for future research. For each member study, there is consent for the study itself, consent for consortium participation and consent for the future uses. These can get muddled with each other, as well as mixed with descriptions of the governance mechanisms being used to protect individuals at each stage. For example, following are examples of anonymised text from two of the prospective consents reviewed. In Table 2, this study asks for consent for cancer research.

Table 2: Consent materials stating local ethics approval for participation in ICGC

\begin{tabular}{|c|c|}
\hline Consent Text & Explanation/Interpretation \\
\hline $\begin{array}{l}\text { "Your tissue will be stored in a freezer and will } \\
\text { be used only by qualified Medical Researchers } \\
\text { for biochemical and genetic studies of cancer. } \\
\text { These studies aim to understand more about what } \\
\text { causes [type of] cancer and why treatment seems } \\
\text { to work for some... but not for others." }\end{array}$ & $\begin{array}{l}\text { Initial research to which } \\
\text { individual is consenting }\end{array}$ \\
\hline $\begin{array}{l}\text { "We may also use your tissue to make long lived } \\
\text { cell lines. Your tissue will be valuable for this } \\
\text { research whether or not you have cancer." }\end{array}$ & $\begin{array}{l}\text { Future research beyond the } \\
\text { immediate study through the } \\
\text { creation of cell lines }\end{array}$ \\
\hline $\begin{array}{l}\text { "These studies have to be approved by the [title] } \\
\text { Ethics Committees at the [Institution] and at the } \\
\text { institution carrying out the research." }\end{array}$ & $\begin{array}{l}\text { Ethics approval for future } \\
\text { research both at site of initial } \\
\text { research (i.e., to participate in } \\
\text { ICGC) and other institutions (i.e., } \\
\text { a researcher wishing to access } \\
\text { data through ICGC) }\end{array}$ \\
\hline $\begin{array}{l}\text { "These bodies abide by the ethical and scientific } \\
\text { principles set out by the [recognised authority] of } \\
\text { [country]." }\end{array}$ & $\begin{array}{l}\text { Use of national ethics and } \\
\text { scientific principles to guide } \\
\text { decision }\end{array}$ \\
\hline
\end{tabular}

Participation in ICGC is included through the mechanism that the local institutional ethics committee will approve any future uses of the materials collected. This differs from the text in Table 3, which specifically states that samples and data will be used within the ICGC project. Future biomedical research is alluded to but not specifically stated. When asked, project leaders from both of these studies, as well as the others who we approached, confirmed that their consents included future biomedical use. 
Table 3: Consent materials including ICGC language

\begin{tabular}{|l|l|}
\hline Consent Text & Explanation/Interpretation \\
\hline $\begin{array}{l}\text { Cancer can result from changes in a person's genetic } \\
\text { material (DNA). By studying the genetic changes, } \\
\text { researchers can learn what causes cancer. This will } \\
\text { lead to new ways to prevent, detect and treat cancer... }\end{array}$ & Initial research \\
\hline $\begin{array}{l}\text { Researchers will study the tumour cells grown...to } \\
\text { learn about the genetic changes in the tumour. Some of } \\
\text { these cells will also be stored for future research. }\end{array}$ & Future research \\
\hline $\begin{array}{l}\text { Scientists around the world are participating in the } \\
\text { ICGC.... In [country], the [institution] is leading a } \\
\text { study on the genetics of [type of] cancer. }\end{array}$ & Participation in ICGC \\
\hline $\begin{array}{l}\text { The ICGC was set up to help researchers investigate } \\
\text { the genetic changes in cancer....The ICGC supports } \\
\text { the sharing of data with the international research } \\
\text { community. This may include researchers from } \\
\text { academia, charitable organizations and the private } \\
\text { sector, such as drug companies.... }\end{array}$ & $\begin{array}{l}\text { Core role of ICGC (cancer } \\
\text { research) and description of } \\
\text { international data sharing }\end{array}$ \\
\hline $\begin{array}{l}\text { Researchers who use your data will sign agreements } \\
\text { that define how your data may be used.... They must } \\
\text { use it only for the purposes to which they agreed with } \\
\text { the ICGC. }\end{array}$ & $\begin{array}{l}\text { Reliance on ICGC } \\
\text { procedures for managing } \\
\text { data access }\end{array}$ \\
\hline
\end{tabular}

\section{Appropriate governance mechanisms are needed to support the consent process}

Consents should aim to be as specific as possible, but not raise inappropriate barriers to research. However, the protection of participants is paramount and the terms of their consents must be honoured. Ambiguous consents for future research are not ideal, but as shown by studies such as the UK Biobank, which successfully reached its target recruitment of 500,000 participants, ${ }^{36}$ people accept this wide ambit, asking in return to have appropriate governance committees and procedures in place to justify their trust. ${ }^{37}$ The ICGC has such mechanisms, such as the Executive Committee and the DACO, to oversee future research uses of participant data. If ambiguities and inconsistencies are suspected and confirmed, steps can be taken that might range from suggesting necessary adjustments that member projects can take, to considering possible sanctions for misuse of data. ${ }^{38}$ In this way, the ICGC will strive to satisfy both the aims of the consortium and the rights of participants.

This confirmation process served several purposes for ICGC and may be applicable to other international consortia. On a practical level, it identified and allowed ICGC to clarify consent issues that might have limited access to ICGC data. In broader terms, it placed responsibility at the local member project level, rather than at the ICGC level, where local and national laws, regulations, ethics oversight and governance bodies can support study leaders and offer protection to participants. It also sent clear 
policy messages to study leaders, member country governments and funders, and the scientific and lay community. The ICGC is committed to providing a resource for cancer research, and related research where possible, and disseminating it to approved researchers across the world, in accordance with the informed consent of its participants.

\section{Conclusion}

The requirement for informed consent is enshrined in bioethical codes of conduct, but how that consent process should be carried out and using what information will vary from study to study. In the case of the ICGC, the member studies represent differing ethico-legal and cultural environments and while this is respected, it is also recognised that without a harmonised process the work of the consortium will not proceed.

Agreement on the activities of the consortium is essential, while acknowledging that the individual studies that are involved may represent different countries and cultures, as well as varying scientific methodologies. Clear principles, flexible guidelines and modifiable model language can help to ensure there is consistency and harmonisation of process. When potential ambiguities arise, a confirmation process can resolve differences.

Some may say that this is not sufficient to protect those who agree to participate in international consortia studies. Perhaps other alternatives for oversight are possible, such as the creation of international bodies to which some governance powers could be delegated by national laws. But if or until such bodies exist, consortia members must work together at the international level to work towards harmonised practices across studies, while relying on national laws, regulatory systems and ethics norms at the local level.

The ICGC is working hard to ensure that all data sets from member projects have the proper consents in place so that research will not be halted or delayed, and the trust of participants will be protected. The ICGC is confident its members will be able to work together towards the common goal of discovering and sharing vital knowledge in the fight against cancer, a goal supported enthusiastically by the participants who have made the research possible. We hope our experiences may benefit other international efforts as well.

\section{Acknowledgements}

The authors wish to thank the ICGC member studies for taking part in our research and members of the ICGC Ethics and Policy Committee for their helpful comments on this manuscript. We also wish to thank all the participants in ICGC member studies.

Funding for this work was provided under a grant from the Ontario Institute of Cancer Research. Portions of this research were presented at the ICGC Ethics and Policy Committee Meeting, $3^{\text {rd }}$ International Cancer Genome Consortium Scientific Workshop, 21-23 March 2010, Madrid, Spain. 
${ }^{1}$ Department of Health Sciences, University of Leicester, Leicester, UK; Centre of Genomics and Policy, Department of Human Genetics, McGill University, Montréal, Canada, Correspondence to: sew40@1e.ac.uk

${ }^{2}$ International HapMap Consortium. The International HapMap Project. Nature, 426, no. 6968 2003: 789-796; 1000 Genomes Project Consortium. A map of human genome variation from population-scale sequencing. Nature 467, no. 7319 2010: 1061-1073.

${ }^{3}$ M. A. Hall, N. M. King, L. H. Perdue, et al. Biobanking, consent, and commercialization in international genetics research: the Type 1 Diabetes Genetics Consortium. Clin Trials 7, no. 1 suppl 2010: S33-S45.

${ }^{4}$ Ibid.

${ }^{5}$ Malaria Genomic Epidemiology Network. A global network for investigating the genomic epidemiology of malaria. Nature 456, no. 7223 2008: 732-737.

${ }^{6}$ Ibid, p. 735.

${ }^{7}$ ICGC. 2008. Scientists Form International Cancer Genome Consortium. http://www.icgc.org/icgc/media/Scientists-Form-International-Cancer-Genome-Consortium (accessed 18 August 2011).

${ }^{8}$ International Cancer Genome Consortium. International network of cancer genome projects. Nature 464, no. 2010: 993-998., p. 993.

${ }^{9}$ Ibid.

${ }^{10} \mathrm{http}: / /$ www.icgc.org.

${ }^{11}$ X. S. Puente, M. Pinyol, V. Quesada, et al. Whole-genome sequencing identifies recurrent mutations in chronic lymphocytic leukaemia. Nature 475, no. 7354 2011: 101-105.

${ }^{12}$ International Cancer Genome Consortium. International Cancer Genome Consortium Goals, Structure, Policies and Guidelines. 2008; http://www.icgc.org/files/icgc/ICGC_April_29_2008_en.pdf (accessed 3 March 2011). p. 13.

${ }^{13}$ Organisation for Economic Co-operation and Development. Guidelines for Human Biobanks and Genetic Research Databases (HBGRDs). http://www.oecd.org/dataoecd/41/47/44054609.pdf (accessed 3 March 2011); Council for International Organizations of Medical Sciences. International Ethical Guidelines for Biomedical Research Involving Human Subjects. 2002;

http://www.cioms.ch/publications/guidelines/guidelines nov 2002 blurb.htm (accessed 3 March 2011).

${ }^{14}$ National Statement on Ethical Conduct in Human Research, Australian Government. 2007.

www.nhmrc.gov.au/ files nhmrc/file/publications/synopses/e72-jul09.pdf (accessed 3 March 2011); Tri-Council Policy Statement. Ethical Conduct for Research Involving Humans. 2010; http://www.pre.ethics.gc.ca/pdf/eng/tcps2/TCPS_2_FINAL_Web.pdf (accessed 14 March 2010); German Ethics Council. Human Biobanks for Research: Opinion. 2010; http://www.ethikrat.org/files/der_opinion human-biobanks.pdf (accessed 3 March 2011). ${ }^{15}$ MalariaGen, op cit 4.

${ }^{16}$ A. Akabayashi, B. T. Slingsby. Informed Consent Revisited: Japan and the U.S. Am J Bioeth 6, no. 1 2006: 9 - 14.; D. A. Chokshi, M. A. Thera, M. Parker, et al. Valid Consent for Genomic Epidemiology in Developing Countries. PLoS Med 4, no. 4 2007: e95.

${ }^{17}$ Editorial. Common consent. Nature 460, no. 7258 2009:933.; S. Conrad, M. Renninger, J. Hennenlotter, et al. Generation of pluripotent stem cells from adult human testis. Nature 460, no. 7258 2009: 1044-1044.; G. Gibson, G. P. Copenhaver. Consent and Internet-Enabled Human Genomics. PLoS Genet 6, no. 6 2010: e1000965.; R. Stein, NIH rejects use of dozens of stem cell colonies by federally funded researchers, in: The Washington Post, Washington, DC, 2010, p. A17.; A. M. Tasse, I. Budin-Ljosne, B. M. Knoppers, J. R. Harris. Retrospective access to data: the ENGAGE consent experience. Eur J Hum Genet 18, no. 7 2010: 741-745.

${ }^{18}$ Ibid.

${ }^{19}$ Conrad, op cit. 17.

${ }^{20}$ Editorial, op cit.17, p. 933.

${ }^{21}$ ICGC, op cit., note 12.

${ }^{22}$ ICGC, op. cit.,note 10. 
${ }^{23}$ Hall, op.cit., note 3 .

${ }^{24}$ ICGC, op. cit., note 8.

${ }^{25}$ ICGC, op.cit., note 11.

${ }^{26}$ J. Kaye, P. Boddington, J. de Vries et al. Ethical implications of the use of whole genome methods in medical research. Eur J Hum Genet 18, no. 4 2010: 398-403; A. L. McGuire, T. Caulfield, M. K. Cho.

Research ethics and the challenge of whole-genome sequencing. Nat Rev Genet 9, no. 2 2008: 152-156.

${ }^{27}$ Wallace, SE. The needle in the haystack - international consortia and the return of individual research results. $J$ Law Med Ethics 2011; 39(4): 631-9.

${ }^{28}$ ICGC, op.cit., note 9.

${ }^{29}$ ICGC, op. cit., note 10 .

${ }^{30}$ Hall, op. cit. 2; A. L. McGuire, L. M. Beskow. Informed Consent in Genomics and Genetic Research. Anпu Rev Genomics Hum Genet 11, no. 1 2010: 361-38.; S. Wallace, S. Lazor, B. M.

Knoppers. Consent and population genomics: The creation of generic tools. IRB, 31, no. 2 2009: 15-20;

The Cancer Genome Atlas. Suggested Informed Consent Language for Prospective Collections. 2011; http://cancergenome.nih.gov/pdfs/6.3.1.1 TCGA.Model.InformedConsent.Form.Prospective Nov2011 508.pdf (accessed 15 March 2012).

${ }^{31}$ Public Population Project in Genomics $\left(\mathrm{P}^{3} \mathrm{G}\right)$. Model Consent Form. http://www.p3gobservatory.org/repository/ethics.htm (accessed 15 March 2012); Public Population Project in Genomics $\left(\mathrm{P}^{3} \mathrm{G}\right)$. Model Information Pamphlet.

http://www.p3gobservatory.org/repository/ethics.htm (accesses 15 March 2012)

${ }^{32}$ International Cancer Genome Consortium. ICGC Research Study Model Consent Brochure (Retrospective/Secondary Use Research).

http://www.icgc.org/files/daco/ICGC_retro_consent_Final.pdf (accessed 17 March 2011).

${ }^{33}$ L. M. Beskow, W. Burke. Offering individual genetic research results: context matters. Sci Trans Med 2010; 2(38): 1-5.

${ }^{34}$ Akabayashi, op. cit., note 16.

${ }^{35}$ Personal communication. The author (SW) is in possession of this email.

${ }^{36}$ UK Biobank. Half a million Britons join world's most detailed health study. 2010. http://www.ukbiobank.ac.uk/wp-content/uploads/2011/07/Recruitment-completed-July-2010.pdf (accessed 15 February 2012).

${ }^{37}$ M. Dixon-Woods, R. E. Ashcroft, C.J. Jackson, et al. Beyond "misunderstanding": Written information and decisions about taking part in a genetic epidemiology study. Soc Sci Med 2007; 65(11): 2212-2222

${ }^{38}$ Y. Joly, N. Zeps, B. Knoppers. Genomic databases access agreements: legal validity and possible sanctions. Hum Genet 2011; 130(3): 441-449. 